\title{
Smac BV6 Has Proapoptotic and Anti-Inflammatory Effects on Rheumatoid Arthritis Fibroblast-Like Synoviocytes Activated by TLR9 Ligand
}

donatella lattuada ( $\boldsymbol{\sim}$ donatella.lattuada@unimi.it)

UNIVERSITY OF STUDY https://orcid.org/0000-0002-0045-3283

Claudia Casnici

Santarelli Foundation

Katia Crotta

University of Milan: Universita degli Studi di Milano

Ornella marelli

University of Milan: Universita degli Studi di Milano

\section{Research Article}

Keywords: rheumatoid arthritis, synovial fibroblasts, smac mimetics, toll-like receptors, apoptosis

Posted Date: May 5th, 2021

DOl: https://doi.org/10.21203/rs.3.rs-474254/v1

License: (c) (i) This work is licensed under a Creative Commons Attribution 4.0 International License. Read Full License 


\section{Abstract}

Rheumatoid arthritis synovial fibroblasts (RASF) are one of the most important cells contributing reumathoid artritis (RA) pathogenesis. The critical role of the Toll-like receptor in inflammation and on autoimmune disease is not fully known. Data rising predominantly from human patients and animal models of autoimmune disease indicate that inappropriate triggering of TLR pathways by exogenous or endogenous ligands may cause the initiation and/or perpetuation of autoimmune reactions and tissue damage. Particularly, the role of TLR9 in RA is still a subject of debate and among TLRs, TLR9 is the only receptor which detects unmethylated CpG motifs in DNA (ODN), and is located intracellularly in endosomes and endoplasmic reticulum. RASFs stimulated by ODN are apoptosis-resistant and contribute to the pathogenesis of RA by producing cytokines and proteolytic enzymes, which degrade the extracellular matrix. Then, we evaluated the proapoptotic and anti-inflammatory activity of the smac BV6 on RASF cultured in synovial fluid (SF), to reproduce the physiopathological environmental characteristics of RA joints. Furthermore, BV6 induces a significant inhibition of the secretion of IL15, TNF alpha, IL6, stimulation of pannus formation, and damage of bone and cartilage in RA. Moreover the secretion of the anti-inflammatory cytokine IL 10 and the cleavage of capase 3 and 8 are dramatically increased in the presence of BV6. Furthermore, TLR9 elicited a robust IFN induction and we reported that in RASF treated with ODN and BV6 the expression of IRF7 is enhanced. Our observations demonstrate that BV6 has beneficial regulatory effects on the inflammatory state induced by TLR9 activation.

\section{Introduction}

Rheumatoid arthritis (RA) is an autoimmune disease characterized by the infiltration and accumulation of activated immune cells in the synovial joints, progressive joint destruction, and functional disability in affected people [1]. Rheumatoid arthritis synovial fibroblasts (RASF) are one of the most important cells contributing to RA pathogenesis and they are potent participants in all aspects of the initiation, progression, and perpetuation of the disease. [2]. The pathognomonic finding of RA is the expansive synovial tissue, called pannus, which erodes cartilage and bone at the cartilage-bone interface and synovial fibroblast resistance to apoptosis and caspase inhibition are a major characteristic of RA. The pannus behaves like a locally invasive tumour and the potential imbalance between the growth and death of RASF is considered a target in the treatment of the disease. Furthermore, RASF can produce local inflammatory cytokines and proteolytic enzymes such as matrix metalloproteinases, which degrade the extracellular matrix, activating osteoclasts resulting in further cartilage degradation and bone erosion [3]. One of the major obstacles to finding a cure for RA is that the factors that drive persistent cellular activation and inflammatory mediator synthesis remain elusive [4]. Many investigators have focused their attention on the molecules of microbial origin in the joints of RA patients that trigger an inflammation by activating various pattern recognition receptors. These receptors namely Toll-like receptors (TLR) are activated by infectious molecular patterns, non-infectious molecular patterns or damage-associated molecular patterns (DAMPs) which includes intracellular molecules as well as extracellular matrix proteins and it has been speculated that aberrant TLR signaling may be involved in the generation of 
autoimmunity [5-9]. There are consistent information from human and animal models supporting a critical role of the TLR/IL1R (interleukin-1 receptor) pathway, at least as a second hit signal in the synovium. Indeed, the TLR pathway amplifies the abnormal crosstalk existing between antigenpresenting cells (APCs), T cells, and B cells, leading to the production of high amounts of proinflammatory cytokines, the expansion of autoreactive lymphocytes. After several months or years, persistent immune activation can lead to RASF hyperplasia, neutrophil recruitment, and complement activation resulting in cartilage destruction, bone erosion, and joint damage [10].

Particularly, the role of TLR9 in RA is still a subject of debate. On the one hand, some studies have proposed that TLR9 worsens the severity of RA. For example, Asagiri et al. showed that the treatment of adjuvant induced arthritic rats with an inhibitor of cathepsin $\mathrm{K}$ led to defective TLR9 signaling and improvement of their pathologic state, even though the role of this protease in the TLR9 signaling pathway is still poorly understood [11,12]. On the other hand, Miles et al. reported that the administration of apoptotic cells in a murine model of collagen-induced arthritis led to a TLR9-dependent antiinflammatory effect, supporting the hypothesis that TLR9 signaling is protective against RA [13]. TLR3 and TLR9 are found primarily in the intracellular endosome and detect internalized ligands. Nucleic acids are readily degraded outside of the cell, however in the RA joint they may be protected by other molecules. Among TLRs, TLR9 is the only receptor which detects unmethylated CpG motifs in DNA and is located intracellularly in endosomes and endoplasmic reticulum [14]. Particularly, TLR9 may directly limit the duration or quality of an immune response through the induction of negative regulators of the TLR7 signaling cascade or other mechanisms that limit the duration of TLR7 activation. Furthermore, the activation of TLR9 induces the production of inflammatory cytokines such as tumor necrosis factoralpha (TNFa), interleukin-6 (IL-6), interleukin-1b (IL-1b) and type1 interferons (IFNs) such as IFNb. TLR9 resides in the endoplasmic reticulum and in order to signal, two distinct pathways are initiated. One is the nuclear factor kB (NF-kB) dependent proinflammatory cytokines pathway, and the other is the interferon regulatory factor 7 (IRF7-dependent type I interferons (IFNs)) pathway [15,16]. Particularly, IRF7 is a multifunctional regulator that participates in immune regulation, cell differentiation, apoptosis, and oncogenesis. Then, TLR9 engagement can culminate in two outcomes: the activation of IRF in the IRFsignaling endosomes (IRF-SE) and the activation of NF- $K$ B in the NF- $K$ B signaling endosomes (NF- $K$ B$\mathrm{SE})$. Thus, the TLR9 signaling pathway has been defined as "bifurcated." [17] Specifically, the trafficking of TLR9 and its ligand to IRF-SE leads to the production of type I IFN, whereas localization to the NF- $K$ B$S E$ induces the expression of proinflammatory cytokines, via IRF and NF- $K B$, respectively [18]. The studies enlarged the signaling function of IAPs in antimicrobial innate immunity by demonstrating their role in signaling pathways initiated by pattern-recognition receptors (PRRs) [19].These include cytosolic nucleotide-binding oligomerization domain containing proteins (NODs) activated in response to intracellular bacterial infection, membrane-bound Toll-like receptors (TLRs) that sensing pathogenassociated molecular patterns (PAMPs) originating from bacteria, viruses, fungi, parasites and cytosolic retinoic acid inducible gene I (RIG I) that recognizes viral RNA. IAPs are recruited to the receptorassociated intracellular signaling complex upon receptor engagement and participate in signal transduction inducing NF-KB and MAPK activation and the expression of proinflammatory genes. [20] 
Thus, human cells possess natural antagonists of IAP-mediated caspase inhibition such as Smac/ DIABLO (a second mitochondria-derived activator of caspases/direct IAP-binding protein with low PI $[21,22])$. Smac mimetics have been developed to target multiple IAPs, including cIAP1, c-IAP2, and XIAP1, inhibiting IAPs, BV6, and similar bivalent IAP antagonists have been shown to induce proteasomal degradation of CIAP1 and CIAP2, abrogate CIAP- and XIAP-mediated inhibition of caspases, and induce TNFa-dependent cell death $[23,24]$. The effects of BV6 have not yet been reported in RA, then we studied how BV6 can turn off the inflammatory activation of TLR9 and investigate its ability to decrease the production of inflammatory cytokines, reduce the recruitment of immune cells at the site of inflammation and therefore could potentially prevent tissue damage caused by chronic inflammatory conditions.

\section{Materials And Methods}

\section{synovial fluid samples and extraction of fibroblast-like synoviocytes}

Synovial fluid samples (SF) and RASF were used. The Ethics Committee at the University of Milan approved the whole study (27th March 2012) and all patients signed informed consent to take part in the study. All samples of liquid and synovial membrane were taken from the waste materials during arthrocentesis or surgery. SF was directly aspirated from the joints of patients, collected into heparinized tubes and centrifuged at $1000 \times g$ for $10 \mathrm{~min}$. The acellular portion was stored at $-80^{\circ} \mathrm{C}$ until use. Samples collected from 10 patients were used to reduce the variability between the different fluids, which were used at a final dilution of 1:8 in culture medium. Synovial tissue was obtained from patients with rheumatoid arthritis $(n=22)$ during joint synovectomies. The tissues were digested with collagenase in Dulbecco's modified Eagle Medium (DMEM, Euroclone, Pero, Italy) for $2 \mathrm{~h}$ at $37^{\circ} \mathrm{C}$. Dissociated cells were then centrifuged at $1000 \times g$, suspended in DMEM supplemented with $10 \%$ opticlone serum (Euroclone, Pero, Italy), $2 \mathrm{mM}$ L-glutamine, 100 units/ml penicillin, and $100 \mu \mathrm{g} / \mathrm{ml}$ streptomycin (Euroclone, Pero, Italy), and plated. Cells were cultured overnight, non-adherent cells were removed, while adherent cells were cultivated in DMEM supplemented with $10 \%$ foetal calf serum at $37^{\circ} \mathrm{C}$ in $5 \% \mathrm{CO}_{2}$. The purity of the cells was tested by flow-cytometric analysis using phycoerythrin-conjugated anti-CD14 (Pharmingen, San Diego, CA, USA), fluorescein isothiocyanate phycoerythrin-conjugated anti-CD3, anti-CD19 and anti-Thy1(CD90) monoclonal antibodies (R\&D Systems Minneapolis, MN). A FacsCalibur flow cytometer (488ex/620em, Becton Dickinson, San Jose, CA, USA) was used for the analysis. At passage 3 , the cells were homogeneous from the morphological point of view and exhibited the characteristic properties (typical bipolar configuration under inverse microscopy) of RASF. Most cells (>98\%) expressed the surface marker for fibroblasts (Thy-1) and were negative for the expression of antigens CD3, CD19, and CD14. Before each experiment, RASF at passages 3-9 were cultured in the presence of SF for 7 days.

\section{TLR expression analysis}

RASF were cultured in the presence of SF for 7 days. To avoid nonspecific binding, the samples were saturated with Odyssey Blocking Buffer (LI-COR Bioscience) for $2 \mathrm{~h}$ with moderate shaking. The plate 
was washed four times with washing solution (0.1\% Tween 20 in PBS) and then incubated for $1 \mathrm{~h}$ with Cell Tag 700CW (1:500; LI-COR Bioscience). Cell Tag 700 stain is a near-infrared fluorescent, nonspecific cell stain that accumulates in the nucleus and cytoplasm of cells and provides an accurate label for cell number counting. After 5 washes, the plate was scanned at $700 \mathrm{~nm}$ using the Odyssey Infrared Imaging System (LI-COR Bioscience).

\section{apoptosis assay}

RASF were cultured in the presence of SF for 7 days and treated with CpG oligonucleotide 2006 (ODN) (InvivoGen, San Diego, USA) $5 \mu \mathrm{M}$ and BV6 (Selleckchem USA) $40 \mu \mathrm{M}$ for 48 hours. Untreated cells were used as negative control. Apoptotic cells were detected with the Annexin V-FITC apoptosis detection kit (Abcam, Cambridge, UK), according to the manufacturer's instructions. All samples were analysed on a FacsCalibur flow cytometer (488ex/620em, Becton Dickinson, San Jose, CA, USA). FITC-conjugated Annexin $\mathrm{V}$ emission was collected in the $\mathrm{FLH}-1$ channel, and propidium iodide ( $\mathrm{Pl}$; for the detection of dead cells) emission was collected in the FLH-3 channel. Data were analysed with Cell Quest software. The percentage of apoptosis was calculated considering cells in both early (Annexin ${ }^{+}, \mathrm{Pl}^{-}$) and late apoptosis (Annexin ${ }^{+}, \mathrm{Pl}^{+}$).

\section{real-time RT-PCR}

After cell treatment, total RNA was purified using Total RNA Purification Plus Micro kit (Norgen Biotek Corporation, Thorold, Canada) and reverse transcribed into cDNA using SuperScript IV VILO Master Mix (Invitrogen), according to the manufacturer's instructions. RT-qPCR was performed using TaqMan Gene Expression Assays (Life Technologies, Italy) and Luna Universal Probe qPCR Master Mix (New England Biolabs), according to the manufacturer's instructions.

GAPDH gene was selected as an internal reference. mRNA expression of IL6, IL15, and TNF alpha, capsase 3,8 , and IRF5 was evaluated. Data were analysed according to the $2^{-\triangle C T}$ method and were normalized by GAPDH expression in each sample.

\section{cytokine and caspases detection}

RASF were cultured in the presence of SF for 7 days and treated for $48 \mathrm{~h}$ with ODN $5 \mu \mathrm{M}$ and BV6 $40 \mu \mathrm{M}$. Cells were lysed in lysis buffer and the protein concentration was measured by the BCA Protein Assay method (Thermo Scientific, USA) according to the manufacturer's instructions. For cytokine detection, the cell lysates were separated by SDS-PAGE in $4-12 \%$ or $10 \%$ Tris-HCl pre-cast gels (Life Technologies, Carlsbad, CA) respectively, and transferred onto nitrocellulose membranes (Life Technologies, Carlsbad, $\mathrm{CA}$ ). The membranes were blocked for $3 \mathrm{~h}$ with $5 \%$ non-fat dry milk (Lab Scientific) in washing medium and probed overnight at $4^{\circ} \mathrm{C}$ with primary antibodies: mouse anti IL6 1:600, anti IL10, 1:1500 (Prospec East Brunswick, NJ USA), anti TNF-a, anti IL15, 1:1000 (from Peprotec, London UK), mouse anti caspase 3 1:500 (Santa Cruz USA) and mouse anti caspase 8 1:1000 (Cell Signalling USA) whereas rabbit anti human ciclophillin, 1:1500 (Immunological Sciences, Italy) was used as the loading control. Secondary antibodies were conjugated to horseradish peroxidase (Bio-Rad laboratories, Milan, Italy ), and the gels 
were developed using Westar $\eta \mathrm{C}$ (Cyanagen, Italy). Images were acquired with the use of ChemiDoc Imaging System apparatus (Bio-Rad Laboratories, Milan, Italy) and analysed with Image Lab ${ }^{\mathrm{TM}}$ software, version 6.0.1 for Windows (Bio-Rad Laboratories, Milan, Italy).

\section{statistical analysis}

Statistical analysis was performed using Student'st test for matched pairs. Differences with a confidence level of $>95 \%$ were considered statistically significant $(p<0.05)$.

\section{Results}

TLRs are expressed on the cell surface or intracellularly in many cell types, especially in cells of the innate immune system, where they function as sensors of infection or damage. Activating TLRs lead to the heightened expression of various inflammatory genes, which have a protective role against infection. Data rising predominantly from human patients and animal models of autoimmune disease indicate that inappropriate triggering of TLR pathways by exogenous or endogenous ligands may cause the initiation and/or perpetuation of autoimmune reactions and tissue damage. Then, to define the expression profile of different TLRs in our cellular model, we analyzed RASF cultured in the presence of SF for seven days and evaluated their expression on these cells. Our results proved that TLR9 is the most expressed on RASF (Fig. 1), and then in our tests we used the TLR9 specific ligand synthetic CpG oligonucleotide analogs containing a $\mathrm{CpG}$ oligodeoxynucleotide (ODN). There is increasingly evidence that TLRs are involved in inflammation and cytokine production, and our previous paper showed that Smac 127, a smac mimetic molecule, induced apoptosis in RASFs [25]; then we studied if smac mimetic BV6 was able to bypass ODN-TLR9 induced apoptosis resistance and turn off the inflammation induced by TLR9. In our hands, BV6 is more powerful than smac 127 in inducing apoptosis (data not shown). Particularly, BV6 and similar bivalent IAP antagonists have been shown to induce proteasomal degradation of cIAP1 and cIAP2, abrogate cIAP- and XIAP-mediated inhibition of caspases, and induce TNFa-dependent cell death [26]. First we compared the ability to induct apoptosis of BV6 with the RASF cultured in medium additioned to SF pool. This pool comes from 10 RA patients, in order to eliminate individual variability and to recreate the physiopathological microenvironment of RA. Furthermore, we extended the time incubation until the seventh day, to recreate a chronic inflammation state in the cell cultures. Five days after the cell plate, the fibroblasts are treated with TLR9 ligand ODN $5 \mu \mathrm{M}$ and BV6 $40 \mu \mathrm{M}$ for 48 hours. To this aim, Annexin V-conjugated FITC and PI staining followed by FACS analysis were used to detect apoptotic cells and, as shown in Fig. 2, BV6 induced significative apoptosis both in ODN stimulated cells (ODN + BV6) and in cells unstimulated (BV6). Then, we evaluated both the mRNA expression of caspases 8 and 3 and the presence of the cultivated form of these caspases in RASF treated with ODN and BV6. Figure $3 \mathrm{~A}$ shows the results of the mRNA expression of caspase 3 (panel left) and caspase 8 (panel right) and, as reported, the activation of TLR 9 induces a significant increase in the gene expression of caspases in the RASF treated with BV6. To confirm our results, we analysed the cleavage of caspases 3 and 8 in the same conditions and, as reported in Fig. 3B, both caspases were cleaved, while the treatment with ODN alone did not induce a significant cleavage of the caspases compared to the CTRL. 
Furthermore, TLR stimulation leads to prompt and tightly regulated inflammatory responses through the induction of pro- and anti-inflammatory cytokines and chemokines, the cytokines are the main part of the immune network to provide communication in RA. After the initial stimuli have occurred, cytokines play a role in the communication between parts of the immune system in every step of the pathophysiology process of RA, then we studied the effects of BV6 on the modulation of cytokines expression on RASF activated by ODN and we explored the BV6 ability to modulate the expression of TNFa, IL6, and IL15 and IL10, cytokines known to be involved in RA pathology. RASF were exposed to SF for seven days in the presence of BV 6 and ODN for 48 hours, then gene expression of these cytokines was quantified by realtime PCR (Fig. 4A). The quantitative evaluation of gene expression clearly showed that RASF chronic exposure and ODN activation improved the mRNA expression of pro-inflammatory cytokines in RASF, while BV6 inhibited the mRNA expression of TNFa, IL6, and IL15 and increased the IL10 production. Simultaneously, we evaluated the production of inflammatory cytokine proteins IL6, IL15, TNFa, and the anti-inflammatory cytokine IL10 by western blot. RASF were cultured in the presence of SF for 7 days, with the final $18 \mathrm{~h}$ in the presence of Golgi inhibitors Brefeldin A and Monensin, to stop cytokine secretion. As reported in Fig. 4B and 4C the TLR9 ligands induced an upregulation of inflammatory cytokines production, while BV6 treatment induced a significant inhibition on the secretion of IL15, IL6 and TNFa, as the production of anti-inflammatory IL10 dramatically increased. Upon ligation with its cognate ligand, TLR9 recruits the MyD88 adapter protein. Subsequently, two distinct pathways are initiated. One is the nuclear factor kB (NF-kB) e dependent proinflammatory cytokines pathway, and the other is the IRF7 dependent type I interferons (IFNs) pathway, so we checked the expression of IRF7 in RASF after treatment with ODN and ODN + BV6. As reported in Fig. 5 in the RASF treated with ODN and BV6, the expression of IRF7 is enhanced, these results are confirmed both by the molecular analysis of the mRNA expression (Fig. 5A) and by the expression of IRF7 (Fig. 5B).

\section{Discussion}

TLRs are expressed on the cell surface or intracellularly in many cell types, especially in cells of the innate immune system, where they act as sensors of infection or damage. Activating TLRs lead to the heightened expression of various inflammatory genes, which have a protective role against infection.

Data rising predominantly from human patients and animal models of autoimmune disease indicate that inappropriate triggering of TLR pathways by exogenous or endogenous ligands may cause the initiation and/or perpetuation of autoimmune reactions and tissue damage. It was reported that TLRs are expressed in the inflamed joints of patients with RA and play an important role in the progression and maintenance of the disease, supporting inflammation and cartilage and bone damage [27]. Here we evidenced that TLR9 is highly expressed in RASF cultured "in vitro" in synovial fluid, to reproduce the physiopathological environmental characteristic of RA joints. To date there are not many published studies investigating the expression and function of TLR9 in synovial fibroblasts, and it is not yet clarified how much synovial fibroblasts can contribute to immune response induced by DNA sensing [28]. Moreover, TLR9 is essential for recognition of microbial CpG DNA or synthetic CpG oligonucleotide analogues containing a $\mathrm{CpG}$ oligodeoxynucleotide (ODN) and TLR9 signalling is initiated by recruitment 
of the adaptor molecule MyD88 followed by the engagement of interleukin (IL)-1R-associated kinases and tumour necrosis factor alpha receptor (TNFR)-associated factor [29], inducing the early activation of NF-kB and production of cytokines such as TNFa [30].

In RA the inhibitory proteins of apoptosis (IAPs) play a fundamental role as are responsible for hyperplasia of synovial fibroblasts and pannus formation at the cartilage-bone interface, where it cloaks the cartilage and erodes the bone [31]. Recently, it has been observed that IAPs play a crucial role in regulation of genes involved in innate and immune immunity, inflammation, survival and cell migration [32]. In particular, the IAPs involvement in innate immunity depends on the modulation of TLRs pathways, that lead to NF-kB transcription, MAP kinase and JNK activation [33].

Smac mimetic compounds are able to induce apoptosis in cancer cells and, unexpectedly, to induce the degradation of IAPs, blocking TLRs activation signals [34]. Thus, we investigated the effect of BV6 on RASF, that are resistant to apoptosis, upon TLR9 activation and we demonstrate that BV6 induces apoptosis not only in RASF cultured in tissue medium but also when TLR9 is activated. Both mRNA and protein of cleaved caspase 3 and caspase 8 were observed in RASF treated with BV6, as well as the proteolytic activation of pro-caspase, to confirm the ability of BV6 to abrogate the IAPs-mediated caspase inhibition. Probably, BV6 induced rapid degradation of clAP-1, cleavage of pro-caspase-3 and caspasedependent cell apoptosis and activated the NF-KB pathway and potentially promoted caspase-dependent and TNFa-induced cell death synergistically [35]. Moreover, the treatment with BV6 inhibits the NF-KB canonical pathway by binding and degrading cIAPs and this prevents the ubiquitylation of RIPK1 and leads to the formation of a complex containing RIPK1, caspase 8, and FADD, which promotes apoptosis. $[35,36]$

There is a substantial evidence that IAPs block cell death induced by TLRs on RASF, but there are only a few reports on the role of IAPs in the regulation of TLR-induced cytokines production.

After TLRs ligation, RASF can secrete more mediators that contribute to the maintenance and perpetuation of the inflammation in RA [16, 37]. It is well known that pro-inflammatory cytokines produced by inflamed synovial tissues in RA such as TNF, IL1, IL6, and IL1, can affect RASF activity and the differentiation of osteoclasts and osteoblasts with effects on bone. In addition, anti-inflammatory cytokines, including IL10, are present in RA joints and inhibit pro-inflammatory cytokines $[38,39]$

Our results show that TLR9 agonist ODN is able to elicit responses mRNA and protein expression for TNFa, IL6 and IL15 and BV6 inhibits this effect while upregulate antinflammatory cytokine IL10 expression. In RA IL10 inhibits pattern recognition receptor signaling through mechanisms, which include downregulation of MyD88 expression [40], and the ubiquitination and subsequent degradation of MyD88dependent signaling molecules such as TRAF6 [41].

Innate pathways can potentially activate viral and stress-inducible gene expression of chemokines and cytokines that promote inflammation, cell recruitment, and joint destruction in RA. These signaling pathways have been implicated in inflammatory arthritis, and the IFN signature induced by innate 
receptor activation has been observed in RA and in addition to the synovium, an IFN profile has been reported in peripheral blood cells of a subset of RA patients (17). One possibility

is that the IFN regulatory factor (IRF) family as IRF7 regulate the IFN response in RA $[27,42,43]$

In RASF untreated IRF7 is weakly express while in the cells treated with ODN we observed an upregulation of IRF7 expression. IRF7 is crucial to induce maximally the expression of IFN- $\alpha / \beta$, indeed, the death domain of MyD88 interacting with IRF7 stimulates the IFN-a promoters and [44]. Our data support the hypothesis that BV6-induced apoptosis is potentiated in ODN-activated RASFs by IRF7 upregulation that leads to apoptosis through the TGF- $\beta$ signal pathway $[45,46]$.

Our data extend knowledge of BV6 and underline the multiple effects on RA of this compound which could make it a valid support for the therapy of diseases where several mediators are involved, such as $\mathrm{RA}$, and in which the simultaneous action on several aspects can be a successful strategy.

\section{Declarations}

\section{Funding}

This study was supported by the Santarelli Foundation.

\section{Conflicts of interest/Competing interests}

The sponsors had no role in the study design, data collection, or analysis; the decision to publish; or the preparation of the manuscript. The authors declare no competing interests.

\section{Availability of data and material}

data transparency

\section{Code availability}

not applicable

\section{Authors' contributions:}

\section{Participated in research design:}

Lattuada Donatella 


\section{Conducted experiments:}

Lattuada Donatella, Casnici Claudia, Crotta Katia

\section{Performed data analysis:}

Lattuada Donatella and Marelli Ornella

\section{Wrote or contributed to the writing of the manuscript:}

Lattuada Donatella, Marelli Ornella, Claudia Casnici,

\section{Ethics approval}

(include appropriate approvals or waivers)

\section{Consent to participate}

Informed consent was obtained from all individual participants included in the study.

\section{Consent for publication}

All authors read and approved the final manuscript.

\section{References}

1. Feldmann M, Maini SR. 2008. Role of cytokines in rheumatoid arthritis: an education in pathophysiology and therapeutics. Immunol Rev 223:719.

2. Han Z, Boyle DL, Chang L, Bennett B, Karin M, et al. 2001. C-Jun N-terminal kinase is required for metalloproteinase expression and joint destruction in inflammatory arthritis. J Clin Invest 108: 7381.

3. Bartok B, Firestein GS. 2010. Fibroblast-like synoviocytes: key effector cells in rheumatoid arthritis. Immunol Rev 233: 233-255.

4. van Vollenhoven RF. 2009. Treatment of rheumatoid arthritis: state of the art. Nat Rev Rheumatol 5:53141.

5. Brentano F, Schorr O, Gay RE, Gay S, Kyburz D. 2005. RNA released from necrotic synovial fluid cells activates rheumatoid arthritis synovial fibroblasts via Toll-like receptor 3. Arthritis Rheum 52: 26562665. 
6. Ultaigh SN, Saber TP, McCormick J, Connolly M, Dellacasagrande J, et al. 2011. Blockade of Toll-like receptor 2 prevents spontaneous cytokine release from rheumatoid arthritis ex vivo synovial explant cultures. Arthritis Res Ther 13: R33.

7. Schnare M, Barton GM, Holt AC, Takeda K, Akira S, Medzhitov R. 2001. Toll-like receptors control activation of adaptive immune responses. Nat Immunol 2: 947-50.

8. Choe JY, Crain B, Wu SR, Corr M. 2003. Interleukin 1 receptor dependence of serum transferred arthritis can be circumvented by Toll-like receptor 4 signaling. J Exp Med 197:537-42.

9. Akira S, Hemmi H. 2003. Recognition of pathogen-associated molecular patterns by TLR family. Immunol Lett 85: 85-95.

10. Trinchieri G, Sher A. 2007. Cooperation of Toll-like receptor signals in innate immune defence. Nat Rev Immunol 7:179-190.

11. Asagiri M, Hirai T, Kunigami T, Kamano S, Gober HJ, Okamoto K, Nishikawa K, Latz E, Golenbock DT, Aoki K, Ohya K, Imai Y, Morishita Y, Miyazono K, Kato S, Saftig P, Takayanagi HCathepsin Kdependent toll-like receptor 9 signaling revealed in experimental arthritis. Science. Feb 1;319 (5863):624-7.

12. Hoffmann MH, Skriner K, Herman S, et al. 2011. Nucleic acid-stimulated antigen-presenting cells trigger $\mathrm{T}$ cells to induce disease in a rat transfer model of inflammatory arthritis. J Autoimmun 36:288-300.

13. Miles K, Heaney J, Sibinska Z, et al. 2012. A tolerogenic role for Toll-like receptor 9 is revealed by Bcell interaction with DNA complexes expressed on apoptotic cells. Proc Natl Acad Sci U S A 109:887892.

14. Takeuchi, S. Akira. 2010. Pattern recognition receptors and inflammation. Cel/ 140 805-820.

15. Hemmi, O. Takeuchi, T. Kawai, et al. 2000. A Toll-like receptor recognizes bacterial DNA. Nature 408 740-745.

16. Akira S, Uematsu S, Takeuchi 0. 2006. Pathogen recognition and innate immunity. Cel/124:783-801.

17. Honda K, Yanai H, Takaoka A, Taniguchi T. 2005. Regulation of the type IIFN induction: a current view. Int Immunol 17:1367e78.

18. Honda K., Yanai, H., Negishi, H. et al. 2005. IRF-7 is the master regulator of type-I interferondependent immune responses. Nature 434:772-778.

19. Hoshino K, Sugiyama T, Matsumoto M, et al. 2006. IkappaB kinase-alpha is critical for interferonalpha production induced by Toll-like receptors 7 and 9. Nature 440:949-953.

20. Vandenabeele P, Bertrand MJ. 2012. The role of the IAP E3 ubiquitin ligases in regulating patternrecognition receptor signalling. Nat Rev Immunol 12: 833-844.

21. Tseng PH, Matsuzawa A, Zhang W, Mino T, Vignali DA et al. 2010. Diff $\square$ erent modes of ubiquitination of the adaptor TRAF3 selectively activate the expression of type I interferons and proinflammatory cytokines. Nat Immunol 11: 70-75. 
22. Verhagen AM, Ekert PG, Pakusch M, et al. 2000. Identification of DIABLO, a mammalian protein that promotes apoptosis by binding to and antagonizing IAP proteins. Cell 102:43-53.

23. Sekimura A, Konishi A, Mizuno K, et al. 2004. Expression of Smac/DIABLO is a novel prognostic marker in lung cancer. Oncol Rep 11:797-802.

24. Sun H, Nikolovska-Coleska Z, Yang CY, et al. 2008. Design of small-molecule peptidic and nonpeptidic Smac mimetics. Acc Chem Res 41:1264-1277.

25. Cossu F, Mastrangelo E, Milani M, et al. 2009. Designing Smac-mimetics as antagonists of XIAP, CIAP1, and cIAP2. Biochem Biophys Res Commun 378:162-167.

26. Lattuada D, Gualtierotti R, Crotta K, Seneci P, Ingegnoli F, Corradini C, Viganò R, Marelli O, Casnici C. 2016. Smac127 Has Proapoptotic and Anti-Inflammatory Effects on Rheumatoid Arthritis FibroblastLike Synoviocytes. Mediators Inflamm 17: 2016:6905678.

27. Brentano F, Kyburz D, Schorr O, Gay R, Gay S 2005. The role of Toll-like receptor signalling in the pathogenesis of arthritis. Cell Immunol 233: 90-96.

28. Ospelt C, Brentano F, Rengel Y, et al. 2008. Overexpression of toll like receptors 3 and 4 in synovial tissue from patients with early rheumatoid arthritis: toll-like receptor expression in early and longstanding arthritis. Arthritis Rheum 58:3684-92.

29. Paludan SR, Bowie AG. 2013. Immune sensing of DNA. Immunity 38:870-80.

30. Akira, S., and K. Takeda. 2004. Toll-like receptor signalling. Rev. Immunol. 4: 499 -511.

31. Kawai T, Sato S, Ishii KJ, Coban C, Hemmi H, Yamamoto M, Terai K, Matsuda M, Inoue J, Uematsu S, Takeuchi O, Akira 2004. Interferon-alpha induction through Toll-like receptors involves a direct interaction of IRF7 with MyD88 and TRAF6. Nat Immunol. Oct;5(10):1061-8.

32. Lopez J, Meier P. 2010. To fight or die - inhibitor of apoptosis proteins at the crossroad of innate immunity and death. Curr Opin Cell Biol; 22: 872-81.

33. Gyrd-Hansen M1, Meier P. 2010. IAPs: from caspase inhibitors to modulators of NF-kappaB, inflammation and cancer. Nat Rev Cancer. Aug;10(8):561-74.

34. Petersen, S.L., L. Wang, and A. Yalcin-Chi. 2007. Autocrine TNF alpha signalling renders human cancer cells susceptible to Smac-mimetic-induced apoptosis. Cancer Cel/12: 445-456.

35. Srinivasula SM, Hegde R, Saleh A, Datta P, Shiozaki E, Chai J et al. 2001. A conserved XIAPinteraction motif in caspase- 9 and Smac/DIABLO regulates caspase activity and apoptosis. Nature 410:112-6.

36. Bertrand, M.J.; Milutinovic, S.; Dickson, K.M.; Ho, W.C.; Boudreault, A.; Durkin, J.; Gillard, J.W.; Jaquith, J.B.; Morris, S.J.; Barker, P.A. 2008. cIAP1 and cIAP2 facilitate cancer cell survival by functioning as E3 ligases that promote RIP1 ubiquitination. Cel/30, 689-700.

37. Mclnnes, I.B., and G. Schett. 2011. The pathogenesis of rheumatoid arthritis. New England Journal of Medicine 365: 2205-2219.

38. Brzustewicz, E. Bryl. 2015.The role of cytokines in the pathogenesis of rheumatoidarthritis Practical and potential application of cytokines as biomarkers and targets of personalized therapy. 
Cytokine 76 527-536.

39. Shaw A.T, Gravallese E.M. 2016. Mediators of inflammation and bone remodeling inrheumatic disease Semin Cell Dev Biol Jan: 49:2-10.

40. Dagvadorj J, Naiki Y, Tumurkhuu G, Hassan F, Islam S, Koide N, Mori I, Yoshida T, Yokochi T. 2008. Interleukin-10 inhibits tumor necrosis factor-alpha production in lipopolysaccharide-stimulated RAW 264.7 cells through reduced MyD88 expression. Innate Immun 14: 109-115.

41. Chang J, Kunkel SL, Chang CH. 2009. Negative regulation of MyD88-dependent signaling by IL-10 in dendritic cells. Proc Natl Acad Sci 106:18327-18332.

42. Sweeney S, Firestein G. 2007. Primer: signal transduction in rheumatic disease--a clinician's guide. Nat Clin Pract Rheumatol3(11):651-60.

43. Malmgaard L. Induction and regulation of IFNs during viral infections. J Interferon Cytokine Res Aug:24(8):439-54.

44. Marie, I., Smith, E., Prakash, A. \& Levy, D.E. 2000. Phosphorylation-induced dimerization of interferon regulatory factor 7 unmasks DNA binding and a bipartite transactivation domain. Cell. Biol. 20, 8803-8814.

45. Carillo MC, Alvarez Mde L, Quiroga AD. 2006. Interferon alfa-2b triggers transforming growth factorbeta-induced apoptosis on preneoplasticliver. Ann Hepatol 5: 244-250.

46. Pokrovskaja K, Panaretakis T, Grander D. 2005. Alternative signaling pathways regulating type I interferoninduced apoptosis. J Interferon Cytokine Res 25:799-810.

\section{Figures}




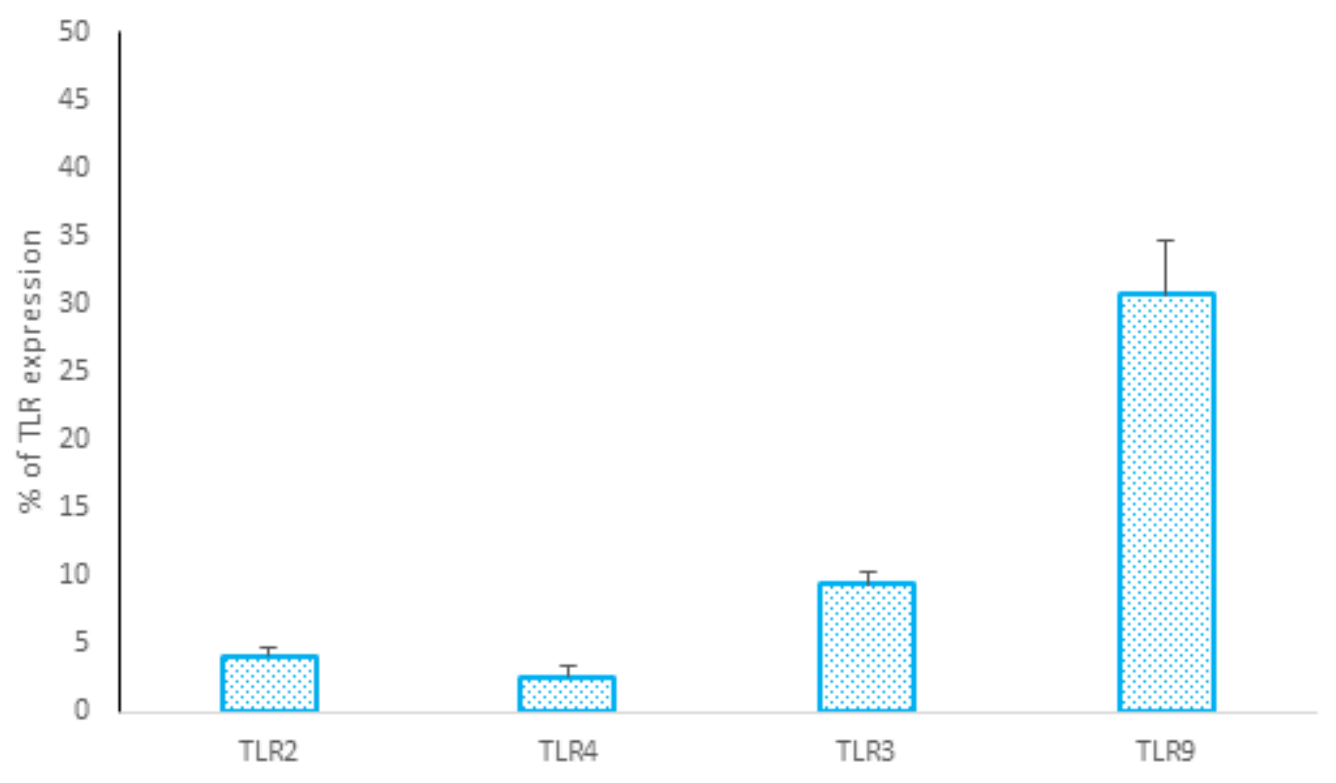

\section{Figure 1}

RASF were cultured in tissue medium in the presence of RA SF pool (1:8 dilution in tissue medium) for 7 days, (CTRL). The TLR expression was performed using Cell Tag 700 Stain is a near-infrared fluorescent, non-specific cell stain that accumulates in the nucleus and cytoplasm of cells and provides an accurate label for cell number counting and the plate was scanned at $700 \mathrm{~nm}$ using the Odyssey Infrared Imaging System. Data are representative of three independent experiments and are shown as means \pm s.e.m of the percentage of TLR expression. 


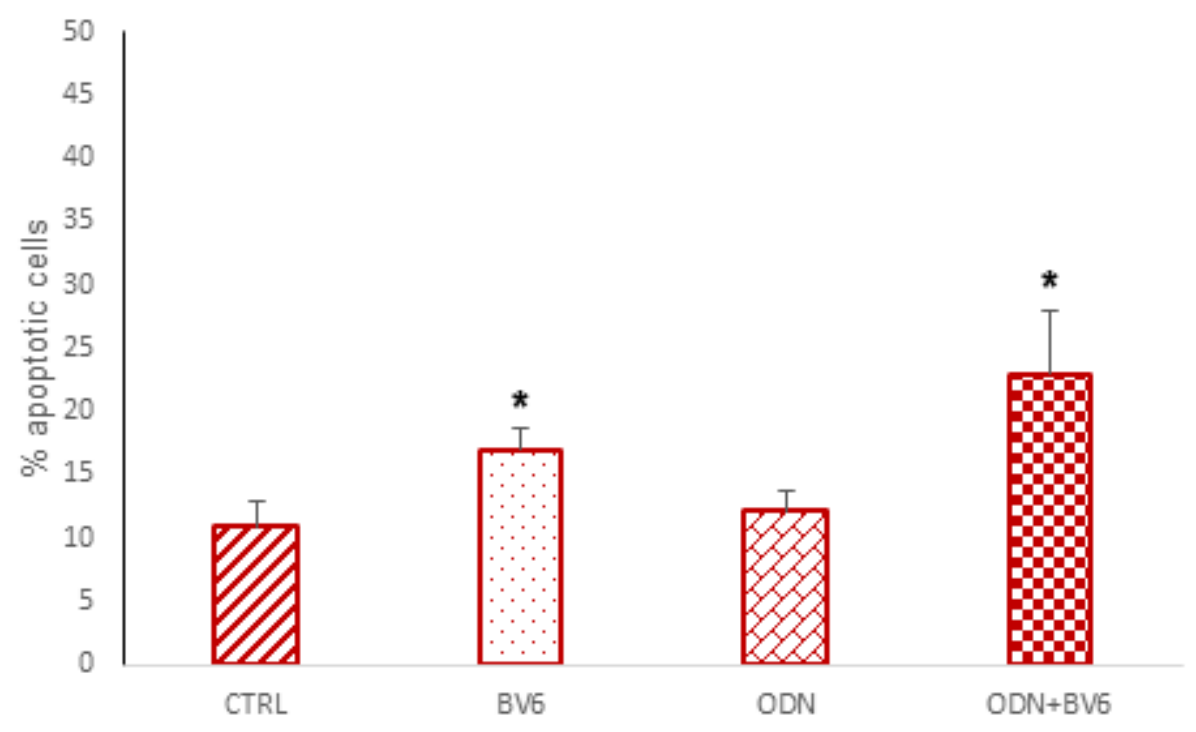

Figure 2

RASF were cultured in the presence of RA SF pool (1:8 dilutions in culture medium) for 7 days, 48 hours before was added ODN $5 \mathrm{\square M}$ and BV6 $40 \mathrm{\square M}$. Quantification of apoptosis was performed by Annexin VFITC conjugate and propidium iodide (PI) staining followed by fluorescence-activated cell sorting (FACS) performed with a FACScalibur flow cytometer equipped with a 488-nm argon laser. The collected data were evaluated by the Cell Quest software. Data were obtained from quadruplicate experiments. Results are shown as means \pm s.e.m. of the percentage of apoptotic cells. CTRL: Cells cultured in medium+SF alone. ${ }^{*} \mathrm{p}<0.05$ vs CTRL. 
A
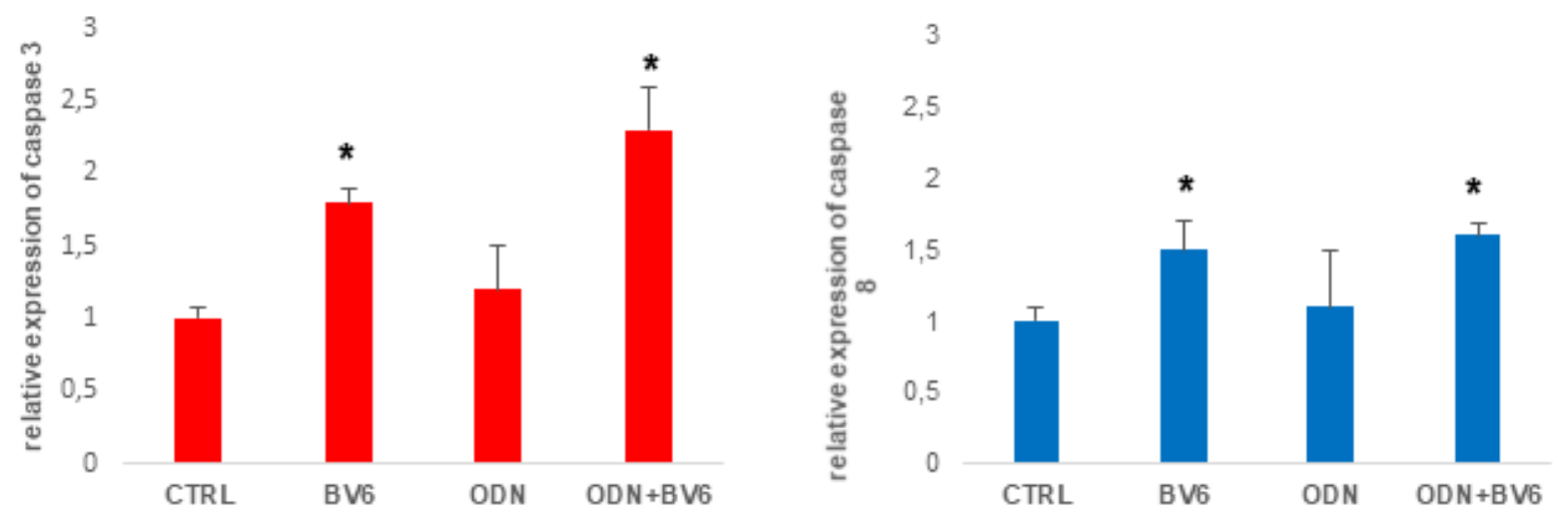

B

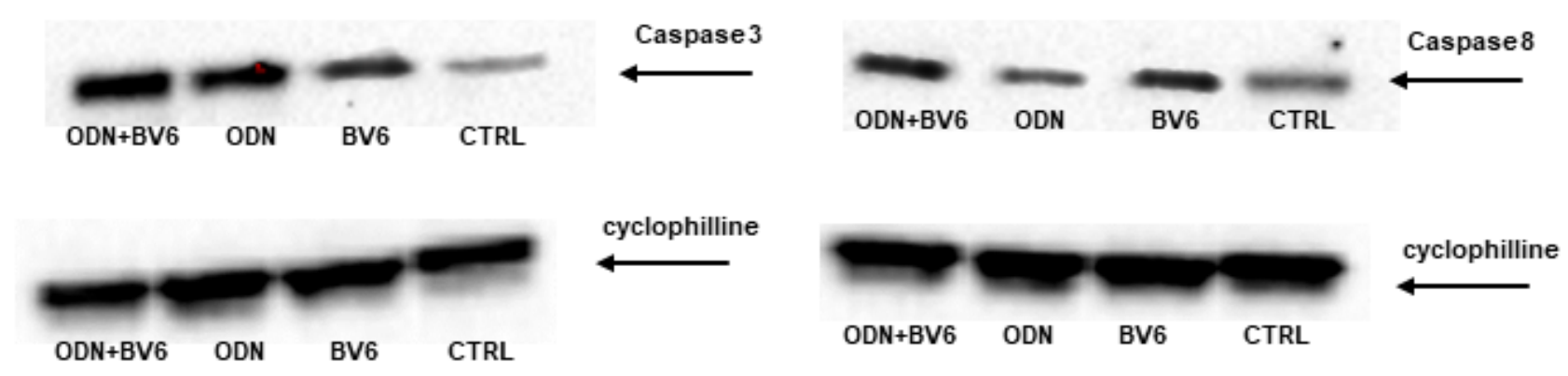

Figure 3

A RASF were cultured in the presence of RA SF pool (1:8 dilutions in culture medium) for 7 days, 48 hours before was added ODN $5 \square \mathrm{M}$ and BV6 $40 \square \mathrm{M}$. Total cellular RNA was extracted with the Total RNA Purification Kit (Norgen Biotek Corp) and reverse transcribed as materials and methods described. Data were analysed according to the comparative Ct method and were normalized by GAPDH expression, according to the equation: 2- $\triangle \mathrm{Ct}[\triangle \mathrm{Ct}=\mathrm{Ct}$ (caspase 3, caspase 8) $-\mathrm{Ct}(\mathrm{GAPDH})]$. Data are expressed as mean $\pm S D$. ${ }^{*} p<0.05$ vs CTRL. B Extracts from RASF treated with RASF cultured in the presence of RA SF pool (1:8 dilution in culture medium) for 7 days, 48 hours before was added ODN $5 \square M$ and BV6 $40 \square \mathrm{M}$, were prepared and levels of caspase 3 and 8 were analysed by western blot. Arrows indicate the mobility of cleaved caspases 3 and 8 ( $18 \mathrm{kDa}$ ). Cyclophylline was used as a loading control (24 kDa).

Densitometric analysis of the immunoblots was performed with Chemidoc Image Lab software (Bio-Rad). Densitometric analysis shows the ratio of caspase/cyclophilline protein expression \pm s.e.m of the mean from four independent experiments. ${ }^{*} p<0.05$ indicates statistically significant difference vs RASF alone 

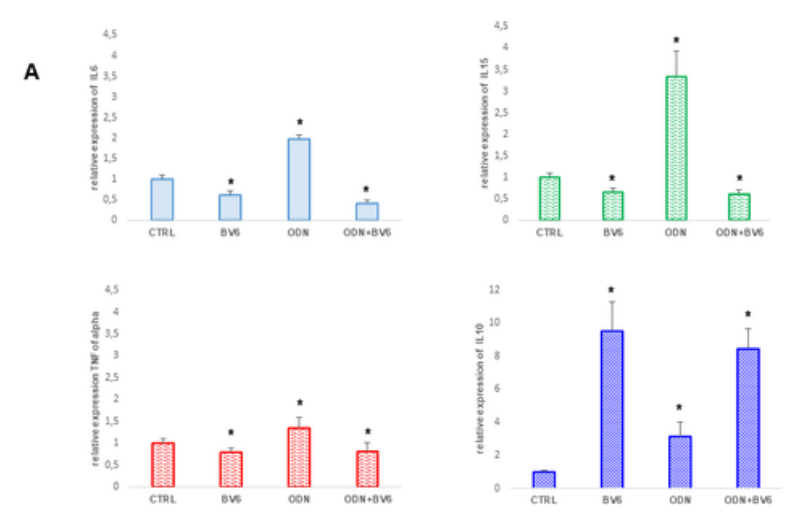

C

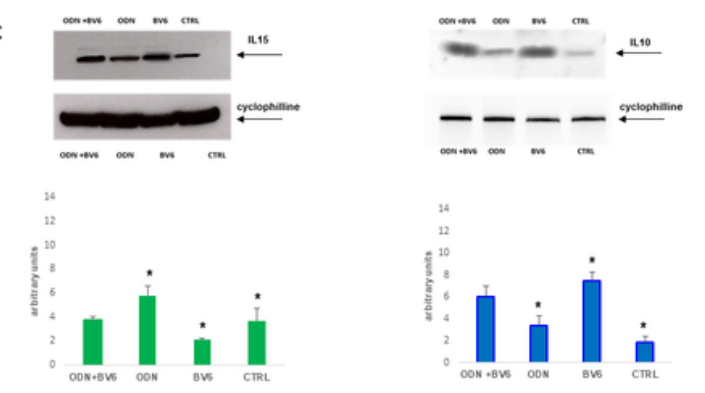

\section{Figure 4}

A RASF were cultured in the presence of RA SF pool (1:8 dilutions in culture mdium) for 7 days, 48 hours before was added ODN $5 \square \mathrm{M}$ and BV6 $40 \square \mathrm{M}$. Total cellular RNA was extracted with the Total RNA Purification Kit (Norgen Biotek Corp) and reverse transcribed as materials and methods described. Data were analysed according to the comparative Ct method and were normalized by GAPDH expression, according to the equation: 2- $\triangle \mathrm{Ct}[\triangle \mathrm{Ct}=\mathrm{Ct}(\mathrm{IL}-6, \mathrm{IL}-15, \mathrm{TNF}-\mathrm{a}, \mathrm{IL}-10)-\mathrm{Ct}(\mathrm{GAPDH})]$. Data are expressed as mean \pm SD. ${ }^{*} p<0.05$ vs CTRL. B Extracts from RASF treated with RASF cultured in the presence of RA SF pool (1:8 dilution in culture medium) for 7 days, 48 hours before was added ODN $5 \square$ M and BV6 $40 \square \mathrm{M}$, were prepared and levels of IL6, TNFQ, IL10 and IL15 (C) were analysed by western blot. Arrows indicate the mobility of the different proteins. Cyclophylline was used as a loading control $(24 \mathrm{kDa})$. Densitometric analysis of the immunoblots was performed with Chemidoc Image Lab software (BioRad). Densitometric analysis shows the ratio of cytokine/cyclophilline protein expression \pm s.e.m of the mean from four independent experiments. ${ }^{*} \mathrm{P}<0.05$ indicates statistically significant difference vs RASF alone 
A
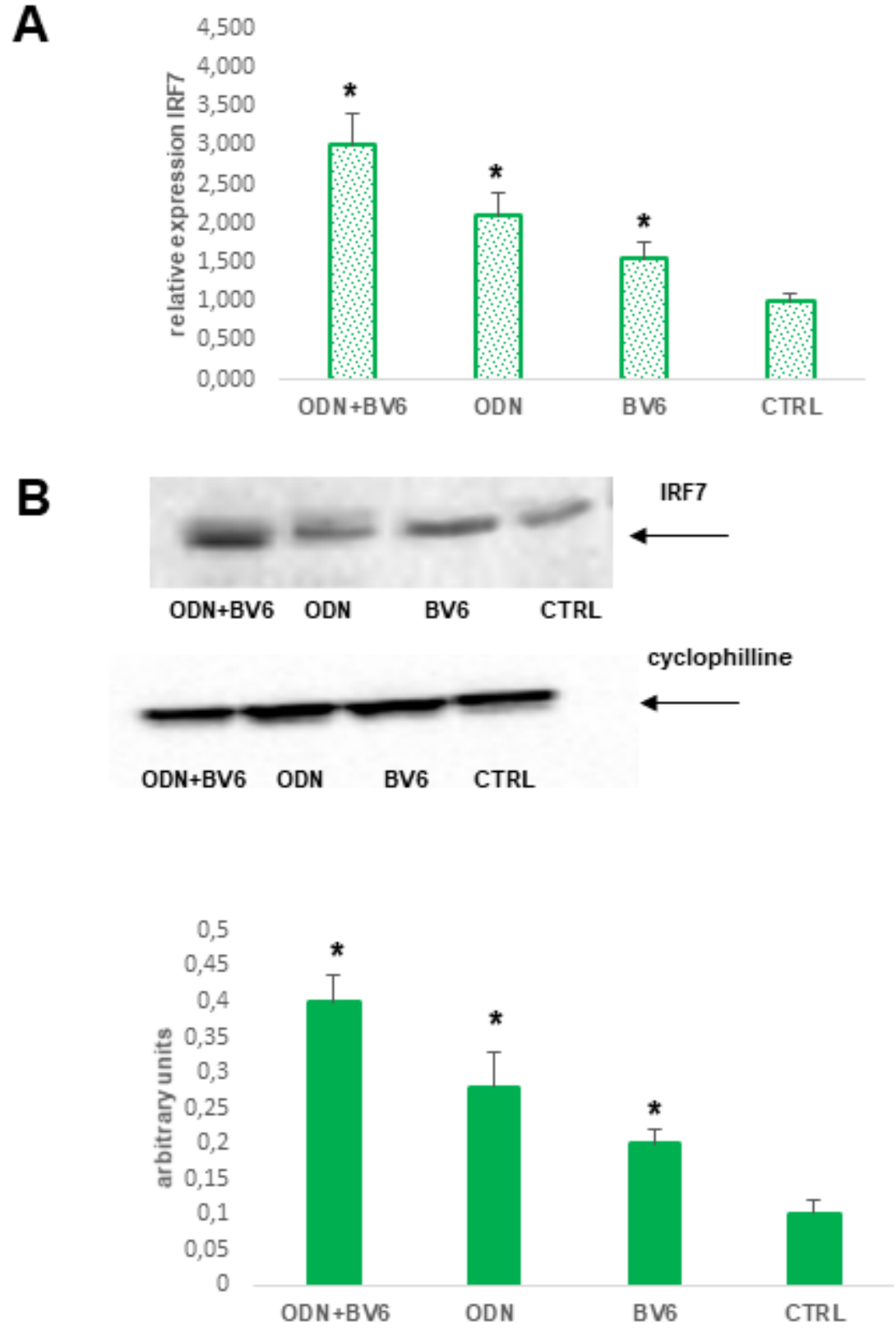

\section{Figure 5}

A RASF were cultured in the presence of RA SF pool (1:8 dilutions in culture medium) for 7 days, 48 hours before was added ODN $5 \square \mathrm{M}$ and BV6 $40 \square \mathrm{M}$. Total cellular RNA was extracted with the Total RNA Purification Kit (Norgen Biotek Corp) and reverse transcribed as materials and methods described. Data were analysed according to the comparative Ct method and were normalized by GAPDH expression, according to the equation: 2- $\triangle \mathrm{Ct}[\triangle \mathrm{Ct}=\mathrm{Ct}(\mathrm{IRF} 5)-\mathrm{Ct}(\mathrm{GAPDH})]$. Data are expressed as mean $\pm \mathrm{SD} .{ }^{*} \mathrm{p}<$ 0.05 vs CTRL. B Extracts from RASF treated as described above were prepared and the levels of IRF7 was analysed by western blot. Arrows indicate the mobility of IRF7 protein. Cyclophylline was used as a 
loading control (24 kDa). Densitometric analysis of the immunoblots was performed with Chemidoc Image Lab software (BioRad). Densitometric analysis shows the ratio of IRF7/cyclophilline protein expression \pm s.e.m of the mean from four independent experiments. ${ }^{*} \mathrm{P}<0.05$ indicates statistically significant difference vs CTRL 